\title{
Transient impact of paclitaxel on mouse fertility and protective effect of gonadotropin-releasing hormone agonist
}

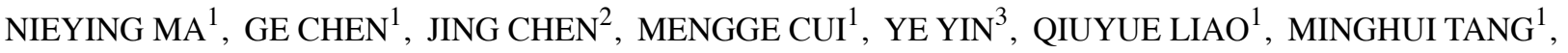 \\ $\mathrm{XUE} \mathrm{FENG}^{1}, \mathrm{XI} \mathrm{LI}^{2}$, SIJIA ZHANG ${ }^{1}$, DING MA ${ }^{2}$, GANG CHEN $^{2}, \mathrm{KEZHEN} \mathrm{LI}^{2}$ and JIHUI AI ${ }^{1}$ \\ ${ }^{1}$ Reproductive Medical Center; Departments of ${ }^{2}$ Gynecological Oncology and ${ }^{3}$ Pediatric Surgery, Tongji Hospital of \\ Tongji Medical College, Huazhong University of Science and Technology, Wuhan, Hubei 430030, P.R. China
}

Received January 4, 2020; Accepted July 13, 2020

DOI: $10.3892 /$ or.2020.7740

\begin{abstract}
Paclitaxel (PXL) is a chemotherapeutic agent widely used in solid tumors. However, whether PXL causes premature ovarian insufficiency in women of reproductive age remains controversial. The aim of the present study was to answer how and for how long PXL affects fertility, and to identify the protective effect of gonadotropin-releasing hormone agonist (GnRHa) in mice. A single dose of PXL was administered to 7-week-old female ICR mice. Mice were treated with GnRHa for 1 estrous cycle prior to chemotherapy, and for another following chemotherapy. On the days 1, 6, 11 and 16 following the administration of PXL, mice were assessed by ovarian histology, ovarian stimulation and mating experiment. Multiple doses of PXL were also administered to verify the duration of the gonadotoxicity of PXL. It was determined that PXL only destroyed antral follicles on day 1 following chemotherapy without reducing primordial follicles. In vitro experiments revealed that PXL impaired oocytes in metaphase, excluding those at the germinal vesicle stage. The number and quality of retrieved metaphaseII(MII) oocytes in PXL-exposed mice were reduced on day 1 following chemotherapy, which was recovered on day 11. MII oocytes from mice pretreated with GnRHa recovered on day 6 following chemotherapy. Following 3 estrous cycles in mice after the last dose of the 3-dose paclitaxel administration, follicles in all stages and retrieved MII oocytes were recovered. It was
\end{abstract}

Correspondence to: Dr Kezhen Li, Department of Gynecological Oncology, Tongji Hospital of Tongji Medical College, Huazhong University of Science and Technology, 1095 Jie Fang Avenue, Wuhan, Hubei 430030, P.R. China

E-mail: tjkeke@126.com

Dr Jihui Ai, Reproductive Medical Center, Tongji Hospital of Tongji Medical College, Huazhong University of Science and Technology, 1095 Jie Fang Avenue, Wuhan, Hubei 430030, P.R. China

E-mail: jihuiai@tjh.tjmu.edu.cn

Key words: ovarian reserve, oocytes, fertility, paclitaxel, gonadotropin-releasing hormone agonist concluded that the impairment caused by PXL on follicles and oocytes in mice lasted for $<3$ estrous cycles, which was shortened by pretreatment of GnRHa.

\section{Introduction}

Cancer is a major public health problem worldwide. Notably, increasing incidences of certain types of cancer, such as cervical (1-3), breast (4) and lung cancer (5), have been reported in young women. Although cancer mortality is continuously declining due to progress in treatment (6), improving cancer-associated outcomes and quality of life in these patients remains imperative. Chemotherapeutic agents are well known for their side effects, such as leukopenia, hepatic insufficiency and premature ovarian insufficiency (7). Endocrine dysfunction and infertility induced by ovarian function impairment in patients of reproductive age can seriously affect their quality of life. The increasing demand for ovarian function preservation has therefore become a major challenge for onco-fertility specialists (8).

According to Information Management System (IMS ${ }^{\mathrm{TM}}$ ) data, the global best-selling chemotherapeutic agent is paclitaxel (PXL) (9), which is widely used in cervical (10), breast (11) and lung cancers (12). Mechanistically, PXL functions as an antineoplastic drug by inhibiting guanosine triphosphate hydrolysis in the microtubule lattice, inducing microtubule stabilization (13). Although PXL had been used for decades, the few studies revealing its effect on the ovaries of animals have had conflicting results (14-18). The results of certain clinical trials and meta-analyses that focused on whether PXL diminishes human fertility were not reliable, due to lack of an accurate ovarian reserve marker (19-22). Therefore, it is important to elucidate how PXL affects ovaries and how long its gonadotoxicity lasts.

Currently, gonadotropin-releasing hormone analogues/agonists (GnRHa) are the first and most widely used agents for ovarian protection during chemotherapy (7). The main mechanism of the protective effect of GnRHa is reducing follicle-stimulating hormone (FSH) levels and suppressing follicle growth to maintain ovaries in a relatively dormant state (7). However, the evidence of fertility preservation by GnRHa remains insufficient, since controversial results were reported in several meta-analyses (23-25). Different 
chemotherapy regimens used in each clinical trial were probably responsible for the contrasting results. It remains unclear whether GnRHa would be effective in protecting ovaries from the gonadotoxicity caused by PXL.

The aim of the present study was to clarify the phenomena and mechanisms through which PXL impairs rodent ovaries, define the duration of its gonadotoxicity and investigate whether GnRHa can protect ovaries during PXL treatment, in the hope of providing laboratory evidence for the clinical application of PXL and GnRHa more safely in women of reproductive age.

\section{Materials and methods}

Animals. Seven-week-old female and 10-week-old male ICR mice were purchased from the Hubei Provincial Center for Disease Control and Prevention, China. Mice were kept for 1 week in the animal husbandry to enable acclimatization to the local conditions in controlled temperature $\left(20-25^{\circ} \mathrm{C}\right)$ and light (12-h light/dark cycle) with free access to food and tap water. Three to four female mice were housed in one ventilated cage with wood shavings as bedding which was changed every 3 days. Each male mouse was separately housed. The whole experiment lasted 3 months, including 501 female mice and 12 male mice. All the animals were monitored every day, and weighed every 5 days. Animals were sacrificed by cervical dislocation for sample collection following inhalation of $70 \% \mathrm{v} / \mathrm{v} \mathrm{CO}_{2}$. Euthanasia was confirmed by the cessation of a heartbeat. Symptoms meeting the NIH guidelines (26) such as abnormal postures, weight loss, loss of appetite or weakness were set as humane endpoints for the present study. All experiments were approved by the Institutional Ethics Committee of Tongji Hospital, Tongji Medical College, Huazhong University of Science and Technology (approval no. TJ-A20161101).

PXL and GnRHa treatment. Female ICR mice, weighing 30-35 g, were randomly assigned to four groups: The vehicle, PXL, GnRHa and PXL+GnRHa groups. Based on our pre-examination and a previous study (27), the estrous cycle of ICR mice was 5 days on average. Therefore, GnRHa $(1 \mathrm{mg} / \mathrm{kg}$, triptorelin acetate; Ferring Pharmaceuticals) or normal saline was administered intraperitoneally to mice prior to chemotherapy for 5 days. Next, animals received a single dose of PXL (30 mg/kg; Pfizer, Inc.) or vehicle intraperitoneally. One quarter of the mice in each group were assessed $24 \mathrm{~h}$ after chemotherapy. The rest of the mice were continuously administered GnRHa for another estrous cycle (5 days) following chemotherapy, and were assessed on days 6,11 and 16 following chemotherapy (Fig. 1A). Another set of mice was administerd $30 \mathrm{mg} / \mathrm{kg}$ PXL every 3 days, for a total of 3 doses. GnRHa (1 mg/kg) was also administered prior to, during and following chemotherapy for a total of 16 days, and mice were then assessed on days 1, 6, 11 and 16 following chemotherapy (Fig. 1B). During the experiment, one mouse in the GnRHa group suffered from weight loss and was euthanized before the endpoint of our experiment.

Histology and follicle count. After anesthetizing by $50 \mathrm{mg} / \mathrm{kg}$ pentobarbital sodium (Merck KGaA) intraperitoneally, blood was collected from the eye orbit for serum anti-müllerian hormone (AMH) analysis (n=5/group/time-point). Then these mice were euthanized by cervical dislocation, and mortality was confirmed by the cessation of a heartbeat. Subsequently, the ovaries were collected. One ovary from each mouse was fixed in $4 \% \mathrm{v} / \mathrm{v}$ paraformaldehyde (Wuhan Servicebio Technology Co., Ltd.) for $24 \mathrm{~h}$ at $4^{\circ} \mathrm{C}$, embedded in paraffin and sectioned at 5- $\mu \mathrm{m}$ thickness for histology. Hematoxylin and eosin (H\&E) staining was performed using standard methods (28). Follicle counts were conducted on serially cut sections from every sixth section of entire ovaries. The follicles were counted at different stages and the mean count per section was calculated for each stage. The follicle stages were classified as previously described (29).

AMH measurement. Blood was collected from the orbit and placed at room temperature for $1 \mathrm{~h}$. Following centrifugation at 3,000 x g, serum AMH levels were determined using a mouse anti-AMH ELISA kit (cat. no. CSB-E13156m; Cusabio Technology LLC), following the manufacturer's instructions.

Oocyte collection and in vitro maturation. An intraperitoneal injection of $10 \mathrm{IU}$ pregnant mare serum gonadotropin (PMSG; Beijing Solarbio Science \& Technology Co., Ltd.) was administered, followed by $12 \mathrm{IU}$ human chorionic gonadotrophin (hCG; Lizhu Pharmaceutical Trading Co., Ltd.) $48 \mathrm{~h}$ after the induction of ovulation in mice. Subsequently, 14-17 h after hCG, cumulus oocytes were collected in an oviduct ampulla after mice were scarified by cervical dislocation following inhalation of $70 \% \mathrm{v} / \mathrm{v} \mathrm{CO}_{2}$. Cumulus cells were removed using $1 \%$ hyaluronidase (Merck KGaA), and denuded metaphase II (MII) oocytes were prepared for further study. In total, 265 mice were sacrificed for oocyte collection.

Oocytes with germinal vesicle (GV) were punctured from ovaries using a $0.5-\mathrm{mm}$ syringe $48 \mathrm{~h}$ following PMSG administration. GV oocytes were cultured in M199 (GE Healthcare Life Sciences) drops under liquid mineral oil (Vitrolife) at $37^{\circ} \mathrm{C}$ in an incubator with $5 \% \mathrm{CO}_{2}$ for in vitro maturation. Oocytes in MI were retrieved after $6 \mathrm{~h}$, and MII oocytes were retrieved after $12 \mathrm{~h}$. GV, MI and MII oocytes were then cultured in $1 \mu \mathrm{M} \mathrm{PXL}$ for $5 \mathrm{~min}$ at $37^{\circ} \mathrm{C}$. GV and MI oocytes were washed for ongoing culture to gain MII oocytes. The ongoing culture time of GV and MI oocytes was 12 and $6 \mathrm{~h}$, respectively. MII oocytes were washed and cultured in normal M199 drops for $4 \mathrm{~h}$ for further study.

Immunofluorescence and confocal microscopy. Oocytes from each group at each time-point $(\mathrm{n}=5)$ were fixed with $4 \% \mathrm{v} / \mathrm{v}$ paraformaldehyde at $4^{\circ} \mathrm{C}$ overnight. They were then transferred to $0.5 \% \mathrm{v} / \mathrm{v}$ Triton X-100 (Beijing Solarbio Science \& Technology Co., Ltd.) for 5 min. Following blocking in $1 \%$ w/v BSA-supplemented (Wuhan Servicebio Technology Co., Ltd.) PBS for $1 \mathrm{~h}$, oocytes were incubated with the monoclonal anti- $\alpha$-tubulin antibody produced in mice at a dilution of 1:1,000 (cat. no. T5168; Merck KGaA) at $4^{\circ} \mathrm{C}$ overnight. Then specimens were then incubated with anti-mouse $\operatorname{IgG}(\mathrm{H}+\mathrm{L})$, $\mathrm{F}\left(\mathrm{ab} \mathrm{b}_{2}\right)_{2}$ Fragment (Alexa Fluor ${ }^{\circledR} 488$ Conjugate) at a dilution of 1:300 (product no. 4408S; Cell Signaling Technology, Inc.) for $1 \mathrm{~h}$ at $37^{\circ} \mathrm{C}$, and then co-stained with Hoechst 33342 (Merck $\mathrm{KGaA}$ ) for $5 \mathrm{~min}$ at $37^{\circ} \mathrm{C}$. Oocytes were mounted onto 
A

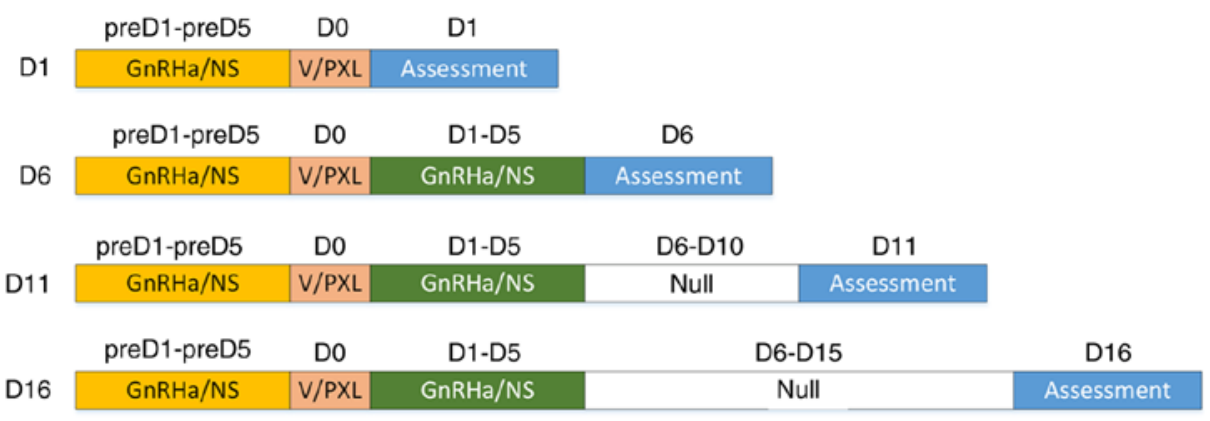

B

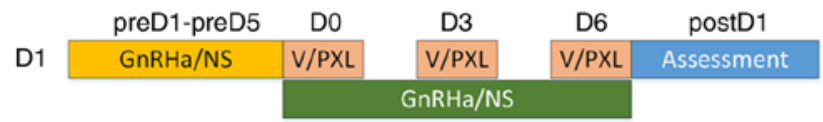

\begin{tabular}{|c|c|c|c|c|c|c|}
\hline \multirow[b]{2}{*}{ D6 } & preD1-preD5 & Do & D3 & D6 & postD1-postD5 & postD6 \\
\hline & GnRHa/NS & V/PXL & V/PXL & V/PXL & GnRHa/NS & Assessment \\
\hline & & & SnRHa/N & & & \\
\hline
\end{tabular}

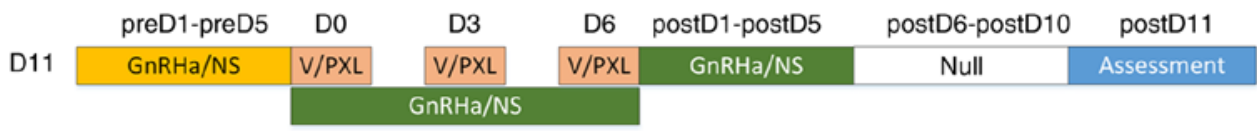

\begin{tabular}{|c|c|c|c|c|c|c|c|}
\hline & preD1-preD5 & DO & D3 & D6 & postD1-postD5 & postD6-postD15 & postD16 \\
\hline D16 & GnRHa/NS & V/PXL & V/PXL & $\mathrm{V} / \mathrm{PXL}$ & GnRHa/NS & Null & Assessment \\
\hline
\end{tabular}

Figure 1. Experimental scheme. (A) Single-dose scheme: Before chemotherapy, mice were pretreated with NS or GnRHa for 5 days (1 estrous cycle). On D0, a single dose of chemotherapy (PXL) or vehicle was administered. On day 1 following chemotherapy, a quarter of the mice were assessed ( $\mathrm{n}=24 / \mathrm{group}$ ). The rest were continuously administered NS or GnRHa for another 5 days. On day 6,11 and 16 following chemotherapy, a quarter of the mice were assessed at each time-point. (B) Multiple-dose scheme: Three doses of paclitaxel were administered consecutively every 3 days and GnRHa was administered continuously during chemotherapy. On days 1, 6, 11 and 16 following chemotherapy, a quarter of the mice (n=10/group) were assessed at each time-point. NS, normal saline; GnRHa, gonadotropin-releasing hormone agonist; PXL, paclitaxel; V, vehicle.

glass slides and observed under a confocal laser-scanning microscope (FV1000; Olympus Corporation).

Chromosome $(\mathrm{CH})$ spread of oocytes. Oocytes from each group at each time-point $(\mathrm{n}=4)$ were placed in hypotonic solution of $0.9 \% \mathrm{w} / \mathrm{v}$ sodium citrate (Wuhan Servicebio Technology Co., Ltd.) for $10 \mathrm{~min}$ at $37^{\circ} \mathrm{C}$ and then exposed to Tyrode's buffer (pH 2.5; Merck KGaA) for $\sim 30 \mathrm{sec}$ at $37^{\circ} \mathrm{C}$ to remove the zona pellucida. Oocytes were then fixed in a drop of $1 \% \mathrm{v} / \mathrm{v}$ paraformaldehyde with $0.15 \% \mathrm{v} / \mathrm{v}$ Triton $\mathrm{X}-100(\mathrm{pH} 9.2)$ on a glass slide. Then slides were dried in a humid chamber for over $2 \mathrm{~h}$ at $37^{\circ} \mathrm{C}$. CHs were stained with $2 \% \mathrm{v} / \mathrm{v}$ Giemsa (Wuhan Servicebio Technology Co., Ltd.) for $10 \mathrm{~min}$ at room temperature and observed under a light microscope at a magnification of x100. The normal number of MII oocyte CHs (univalents) was 20 . Aneuploidy oocytes had \pm 20 univalents.

In vitro fertilization. The caudae epididymides of healthy 12 -week-old male mice scarified by cervical dislocation following inhalation of $70 \% \mathrm{v} / \mathrm{v} \mathrm{CO}_{2}$ were lanced in G-IVF medium (Vitrolife) to release sperm. Following capacitation, sperm was added to pooled oocytes from 4 mice in each group, at each time-point, in G-IVF medium, for $5 \mathrm{~h}$ at $37^{\circ} \mathrm{C}$ and $5 \% \mathrm{CO}_{2}$. These oocytes were then moved to drops of $\mathrm{G} 1$ (Vitrolife) medium under mineral oil. The presence of two pronuclei was considered as successful fertilization.
Mating protocol. Six female mice in the estrous cycle from each group at each time-point mated with healthy male mice which had been demonstrated to be fertile at a ratio of $3: 1$ for $72 \mathrm{~h}$. No special treatment was administered to male mice. Female mice were separated as soon as a plug was observed or after $72 \mathrm{~h}$ of mating.

Pup CH analysis. Three pups from each pregnant mouse were selected randomly for $\mathrm{CH}$ analysis. Two-week-old pups were intraperitoneally injected with $0.5 \% \mathrm{w} / \mathrm{v} 0.1 \mathrm{ml}$ colchicine (Jialin) and sacrificed by cervical dislocation following inhalation of $70 \% \mathrm{v} / \mathrm{v} \mathrm{CO}_{2} 20 \mathrm{~min}$ after injection at room temperature. Bone marrow from the thighbones was flushed into 0.075 M potassium chloride (Wuhan Servicebio Technology Co., Ltd.) solution, and then, marrow cells were fixed with methanol-acetic acid (3:1) fixative for $30 \mathrm{~min}$ at room temperature. $\mathrm{CH}$ slides were conventionally stained with $2 \% \mathrm{v} / \mathrm{v}$ Giemsa solution for $10 \mathrm{~min}$ at room temperature. The number of $\mathrm{CHs}$ was counted under a light microscope at magnification of x100 to determine aneuploidy.

Statistical analysis. The results are presented as the mean \pm SEM. The data were analyzed by one-way ANOVA followed by LSD post hoc or $\chi^{2}$ test using SPSS 17.0 (SPSS, Inc.). A P-value of $<0.05$ was considered to indicate a statistically significant difference. 
A
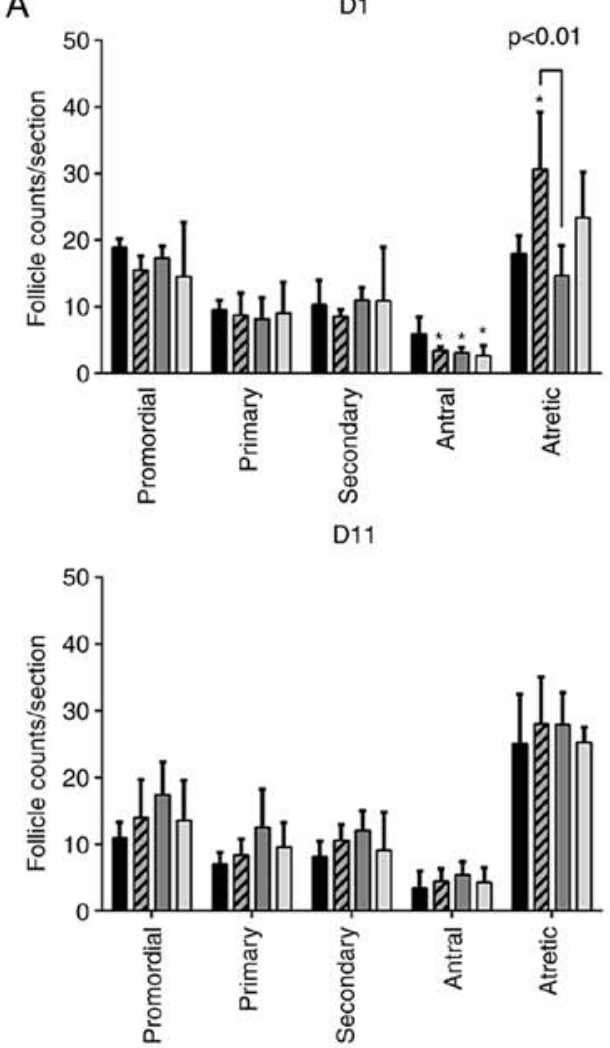

D6
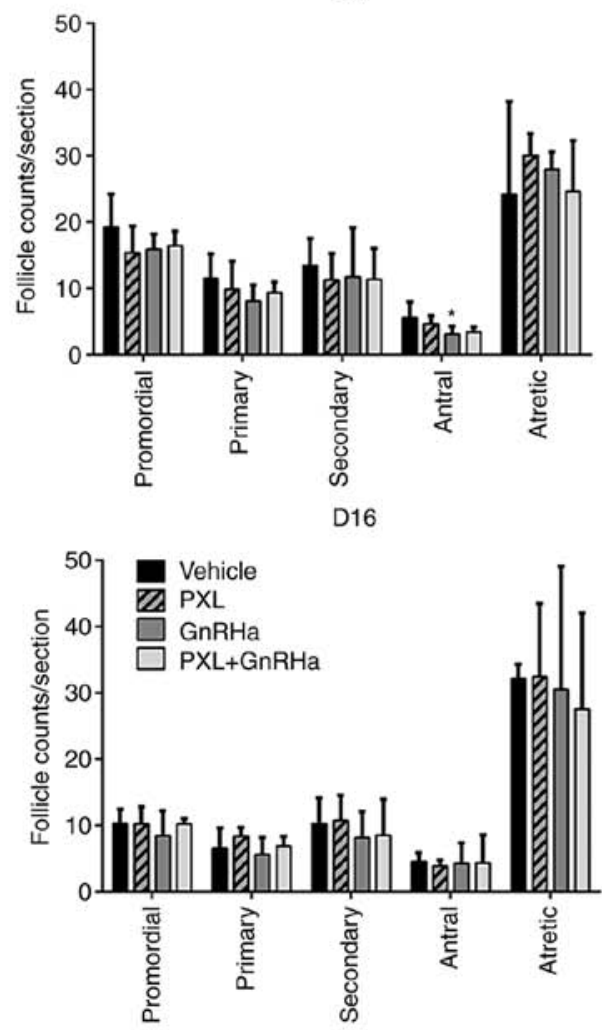

B

PXL

$\mathrm{GnRHa}$

$\mathrm{PXL}+\mathrm{G} n \mathrm{RHa}$
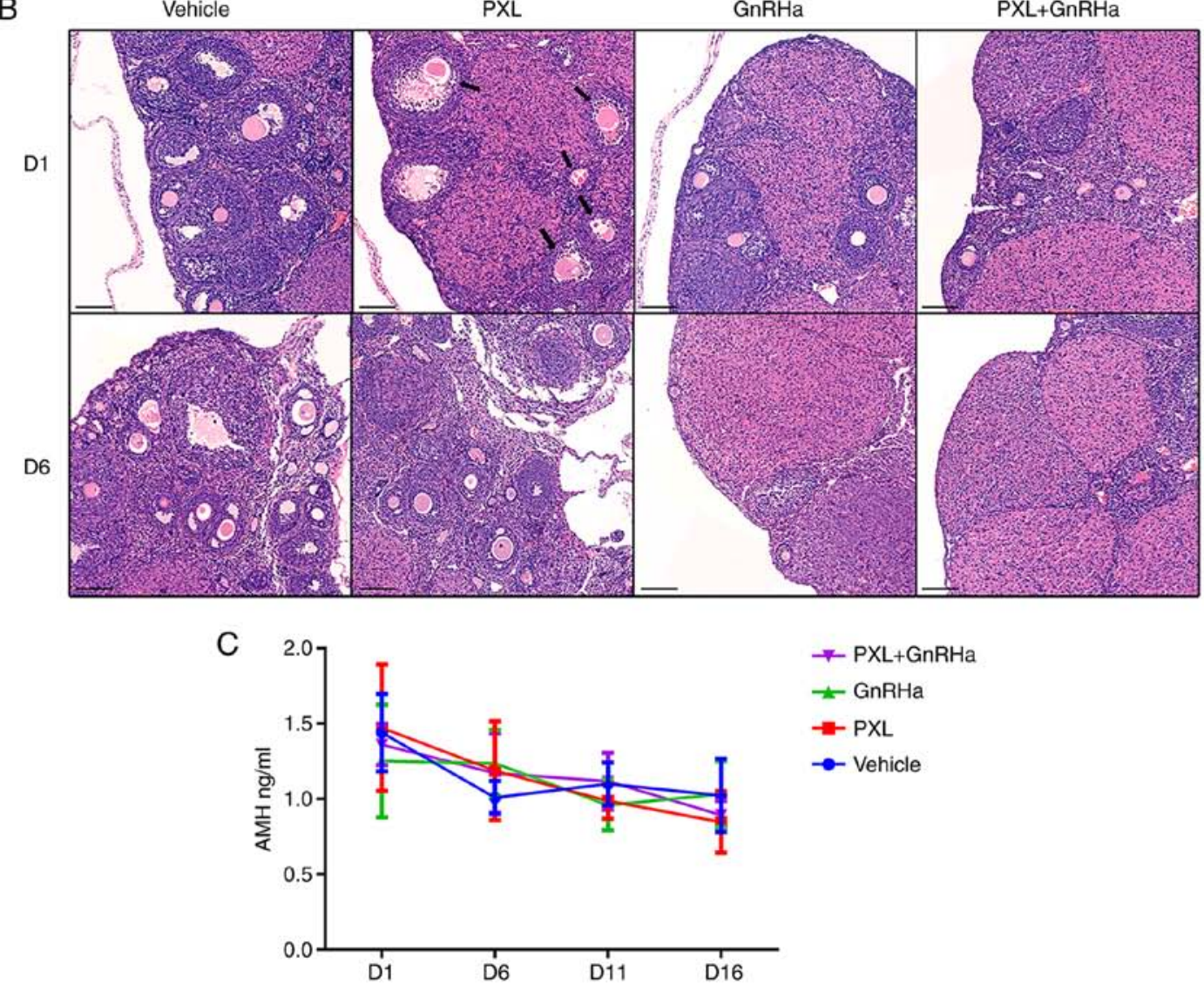

Figure 2. Damage caused by PXL on follicles only lasts for 1 estrous cycle, and GnRHa tends to abrogate this damage. (A) Number of primordial, primary, secondary, antral and atretic follicles on days 1-16 following chemotherapy. Data are expressed as the mean \pm SEM. Statistical analysis was performed by one-way ANOVA followed by LSD test. ${ }^{*} \mathrm{P}<0.05$ vs. the control group (vehicle). (B) H\&E-stained sections revealing representative histological fields in the four groups on days 1-6 following chemotherapy (original magnification, x10). The arrows indicate atretic follicles, which were mainly from antral follicles. Scale bars, $100 \mu \mathrm{m}$. (C) Serum AMH levels in the 4 groups on day 1-16 following chemotherapy. Data are expressed as the mean \pm SEM. PXL, placlitaxel; GnRHa, gonadotropin-releasing hormone agonist; H\&E, hematoxylin and eosin; AMH, anti-müllerian hormone. 

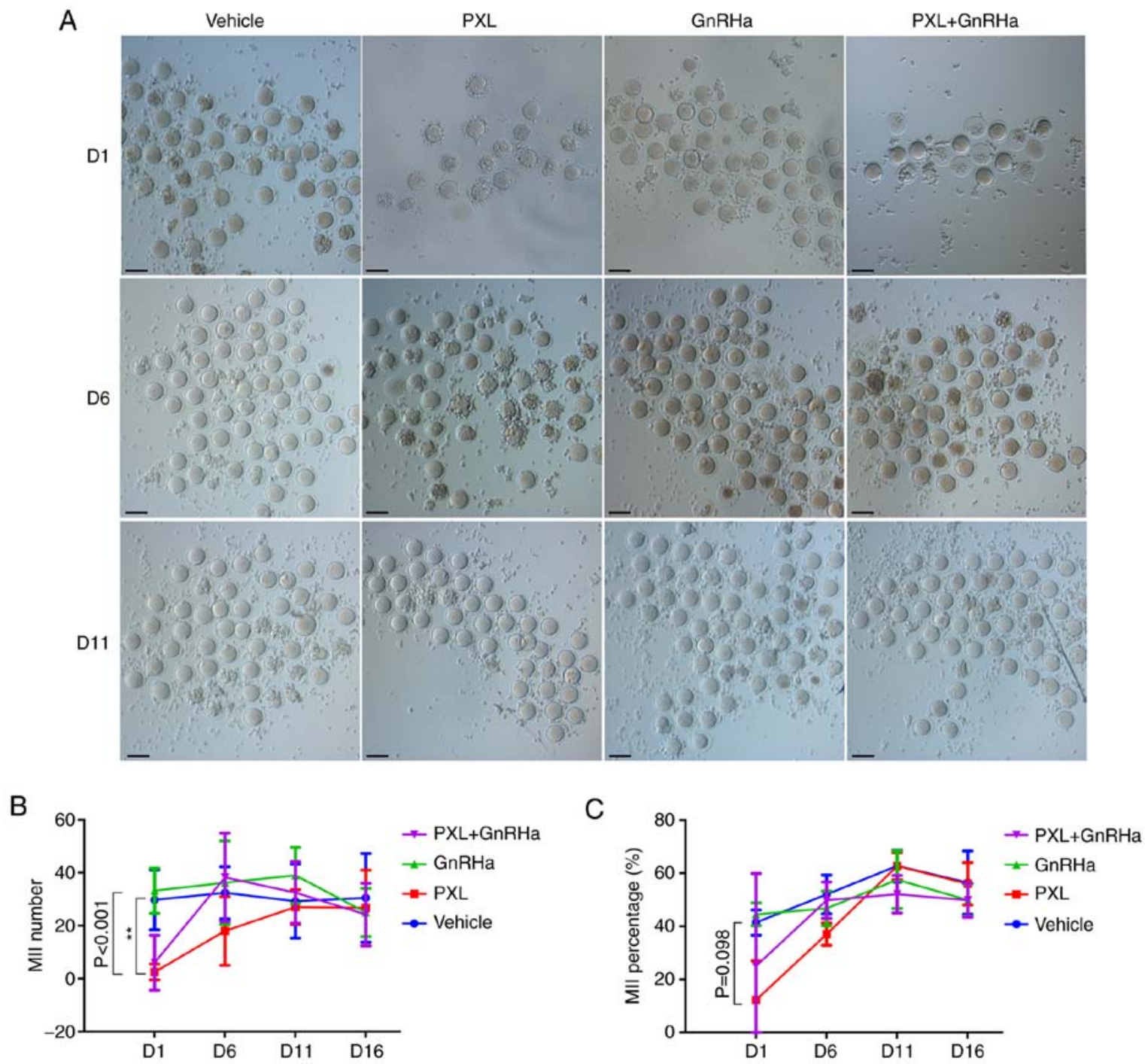

Figure 3. PXL-induced reduction of MII oocytes lasts for <2 estrous cycles, and GnRHa shortens the recovery time. (A) Oocyte morphology under a stereomicroscope (original magnification, x10). Scale bars, $100 \mu \mathrm{m}$. (B) The number of oocytes in the four groups on days 1-16 following chemotherapy. Data are expressed as the mean \pm SEM. (C) MII percentage in the four groups on days 1-16 following chemotherapy. Data are expressed as the mean \pm SEM. Statistical analyses was performed by one-way ANOVA followed by LSD post hoc test. ${ }^{* *} \mathrm{P}<0.005$ vs. the control group (vehicle). PXL, paclitaxel; MII, metaphase II; GnRHa, gonadotropin-releasing hormone agonist.

\section{Results}

PXL only damages antral follicles in a transient manner and does not jeopardize ovarian reserve. In order to determine the impact of PXL on ovarian reserve, histological analysis of the ovaries was performed and the serum AMH of mice was evaluated. On day 1 following the injection of PXL, only antral follicles were significantly reduced in the PXL group, as compared with the control group (3.35 \pm 0.30 vs. $5.90 \pm 1.27$; $\mathrm{P}<0.05)$. The antral follicles in the GnRHa group $(3.07 \pm 0.47$ vs. $5.90 \pm 1.27 ; \mathrm{P}<0.05)$ and $\mathrm{PXL}+\mathrm{GnRHa}(2.60 \pm 0.75$ vs. $5.90 \pm 1.27 ; \mathrm{P}<0.05)$ groups were also reduced, due to the suppressing effect of GnRHa. Atretic follicles were significantly increased in the PXL group as compared with the control group $(30.67 \pm 4.27$ vs. $17.95 \pm 1.35 ; \mathrm{P}<0.01)$. Co-administration of GnRHa plus PXL tended to abrogate the increase of atretic follicles caused by PXL, but not significantly $(23.35 \pm 3.44$ vs. $30.67 \pm 4.27 ; \mathrm{P}=0.09$ ). Primordial, primary and secondary follicles were compared between groups (Fig. 2A and B).
After 1 estrous cycle, on day 6 after chemotherapy, the follicle counts between the four groups had already exhibited no difference in each follicular stage, except that antral follicles in the GnRHa group were still less than the control $(3.10 \pm 1.09$ vs. 5.60 $\pm 1.53, \mathrm{P}<0.05$ ) (Fig. 2A). On days 11 and 16 following chemotherapy, there was no difference in follicle counts between the four groups. Histological analysis revealed that PXL destroyed antral follicles in a transient way and had no effect on primordial follicles. To further confirm the mild effect of PXL on ovarian reserve, serum AMH was assessed in mice, and the result revealed a stationary trend of AMH without any significant difference between the groups at each time-point (Fig. 2C).

PXL induces meiotic arrest in oocytes in metaphase but not in $G V$ stage in vitro. The antral follicles were damaged by PXL where oocytes resumed meiosis, experienced germinal vesicle breakdown (GVBD) and $\mathrm{M} \mathrm{I}$, and then stopped at MII, waiting for fertilization. Following exposure to PXL and 
A

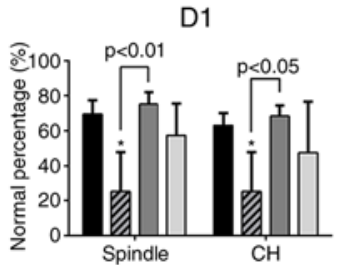

B

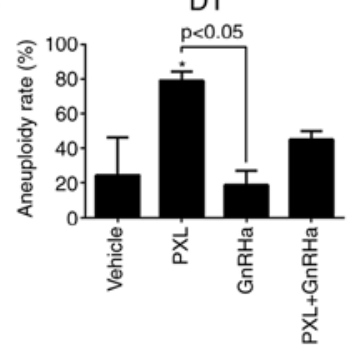

C

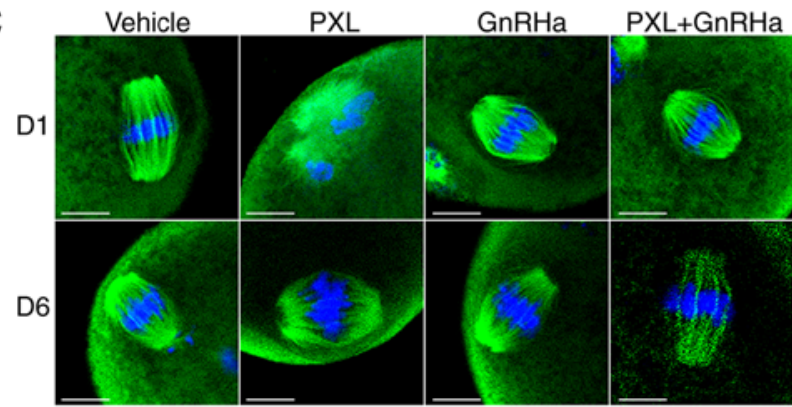

D6

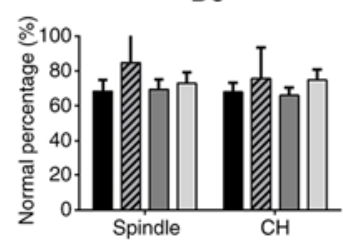

D6

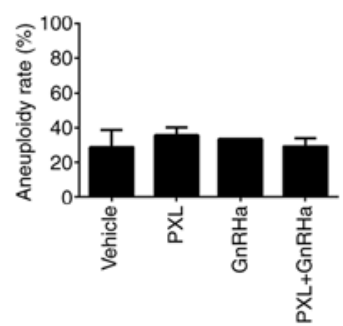

D11

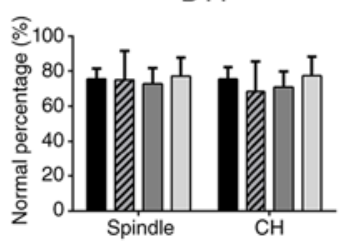

D11

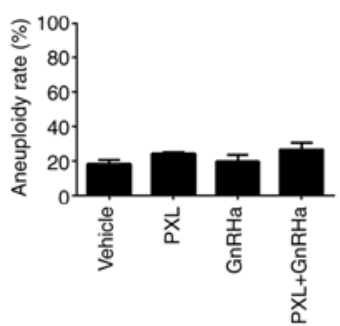

D16

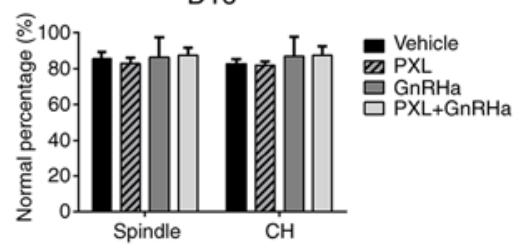

D16
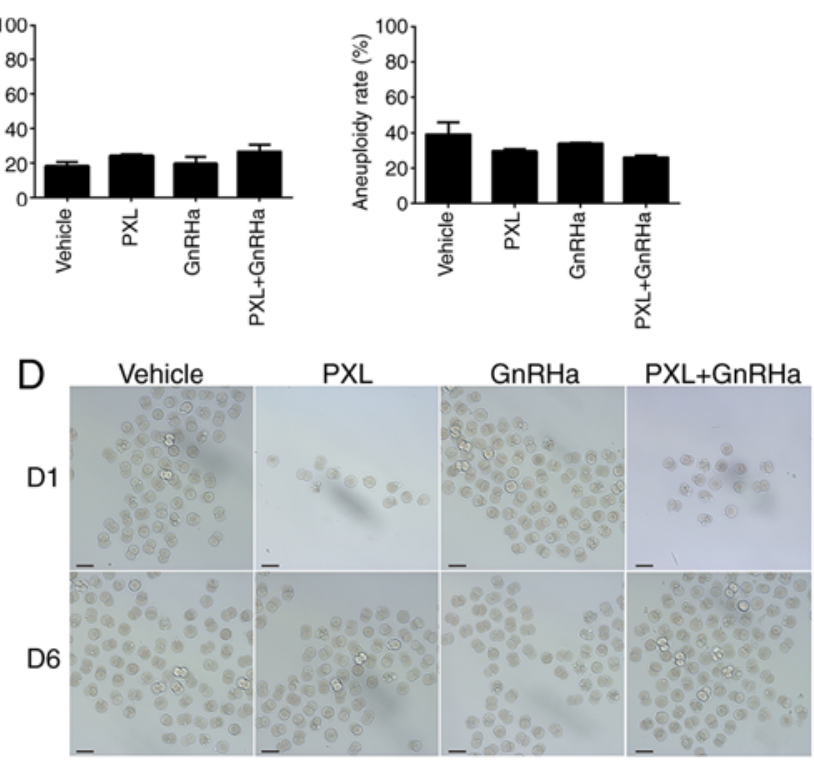

Figure 4. PXL-induced impairment of MII oocytes lasts for 1 estrous cycle, and GnRHa tends to protect against it. (A) Percentage of oocytes with a normal spindle morphology and aligned CHs. (B) Aneuploid rate of oocytes following oocyte $\mathrm{CH}$ spread. Data are expressed as the mean \pm SEM. Statistical analysis was performed by one-way ANOVA followed by LSD test. * $\mathrm{P}<0.05$ vs. the control group (vehicle). (C) Representative images of spindles and CHs in MII oocytes. CHs are shown in blue and microtubules in green under a confocal microscope (original magnification, x100). Scale bars, $5 \mu$ m. (D) Embryos at the 2-cell stage $12 \mathrm{~h}$ after fertilization under a stereomicroscope (original magnification, x10). Scale bars, $100 \mu \mathrm{m}$. PXL, placlitaxel; MII, metaphase II; GnRHa, gonadotropin-releasing hormone agonist; $\mathrm{CH}$, chromosome.

washing, GV oocytes were continuously cultured. The results revealed that PXL did not affect the GVBD $(75.17 \pm 1.81 \%$ vs. $77.45 \pm 2.85 \% ; \mathrm{P}>0.05$; Fig. $\mathrm{S} 1 \mathrm{~A})$ or maturation rate $(43.65 \pm 4.81 \%$ vs. $41.02 \pm 4.13 \%$; P>0.05; Fig. S1A), which suggested that GV oocytes were either away from or resistant to the damage produced by PXL. MI oocytes were also administered PXL, which was then washed off. Following ongoing maturation, the oocytes exposed to PXL barely extruded the polar body, and the maturation rate was markedly lower $(27.84 \pm 10.00 \%$ vs. $86.80 \pm 4.40 \%, \mathrm{P}<0.005$; Fig. S1B). Immunostaining revealed that the spindle apparatuses of these arrested MI oocytes that were exposed to PXL were mostly damaged, while the majority of MI oocytes without PXL administration exhibited normal spindle organization and $\mathrm{CH}$ alignment (spindle, $3.70 \pm 11.33 \%$ vs. $69.84 \pm 20.78 \%$, $\mathrm{P}<0.05$; CH, $0.00 \pm 0.00 \%$ vs. $73.01 \pm 27.74 \%$; $\mathrm{P}<0.05$; Fig. S1B). Similarly, PXL-exposed MII oocytes had a clearly disordered spindle organization and $\mathrm{CH}$ alignment, as compared with the controls (spindle, $29.72 \pm 3.47 \%$ vs. $71.28 \% \pm 17.99 ; \mathrm{CH}$, $26.62 \pm 6.50 \%$ vs. $60.72 \pm 14.97 \%$; $\mathrm{P}<0.05$; Fig. S1C). As a result, the fertilization rate of PXL-exposed MII oocytes was markedly reduced $(9.49 \pm 6.41 \%$ vs. $46.35 \pm 8.72 \%, \mathrm{P}<0.01$; Fig. S1C), while nearly half of the control oocytes were able to be fertilized and develop into 2-cell embryos. It was confirmed that only oocytes in metaphase and not earlier-stage oocytes were affected by PXL through the destruction of spindle apparatuses.

PXL-induced impairment of MII oocytes lasts for 2 estrous cycles in vivo, and GnRHa can protect oocytes. To confirm the effect of PXL on mouse oocytes in vivo, the amount and morphology of oocytes retrieved following ovarian stimulation in mice was observed. On day 1 following chemotherapy, retrieved MII oocytes in the PXL group were significantly less than those in the vehicle group $(2.50 \pm 1.50$ vs. $29.75 \pm 5.65$; $\mathrm{P}<0.005$ Fig. 3B), and the percentage of MII in retrieved oocytes had also decreased $(12.22 \pm 14.8 \%$ vs. $41.40 \pm 4.7 \%$; $\mathrm{P}=0.096$; Fig. 3C). At this time-point, GnRHa did not rescue the number of PXL-exposed oocytes, since the MII oocytes retrieved in the PXL+GnRHa group were not more than those retrieved in the PXL group (6.0 06.0 vs. $2.50 \pm 1.50 ; \mathrm{P}>0.05)$. On day 6 following chemotherapy, the MII oocyte number and percentage in the PXL group started to recover, but was

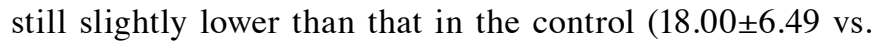

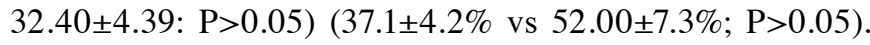
In addition, mice co-treated with GnRHa took less time to completely recover. In the PXL+GnRHa group, the number and percentage of MII oocytes were totally similar to those of the controls $(38.25 \pm 8.35$ vs. $32.40 \pm 4.39 ; \mathrm{P}>0.05)(49.85 \pm 6.9 \%$ vs. $52.00 \pm 7.3 \%$; P>0.05), and the number of MII oocytes was 
A

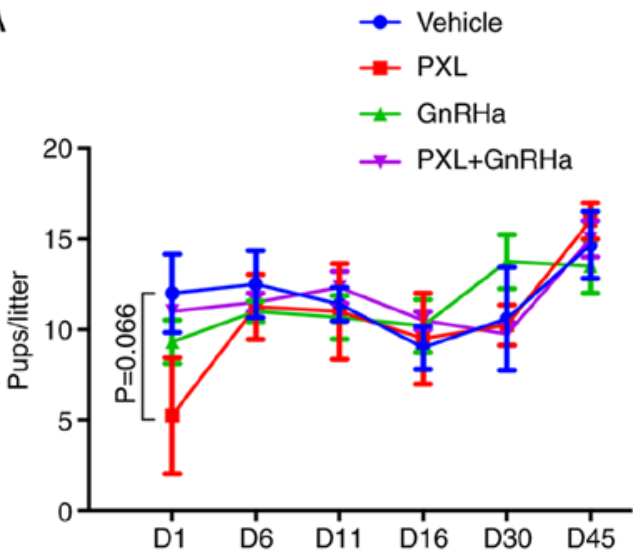

C

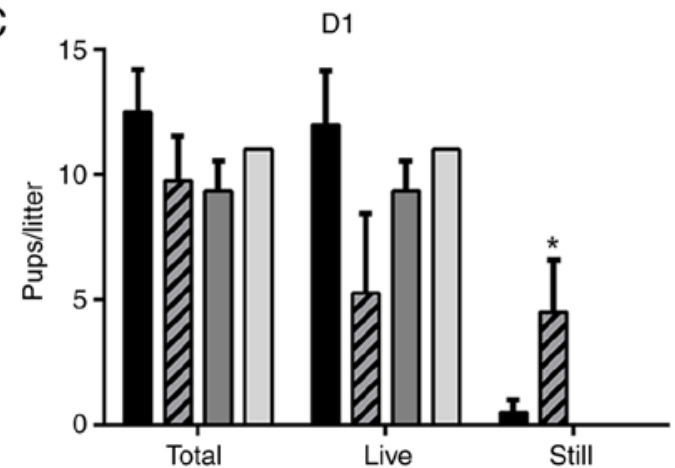

D11

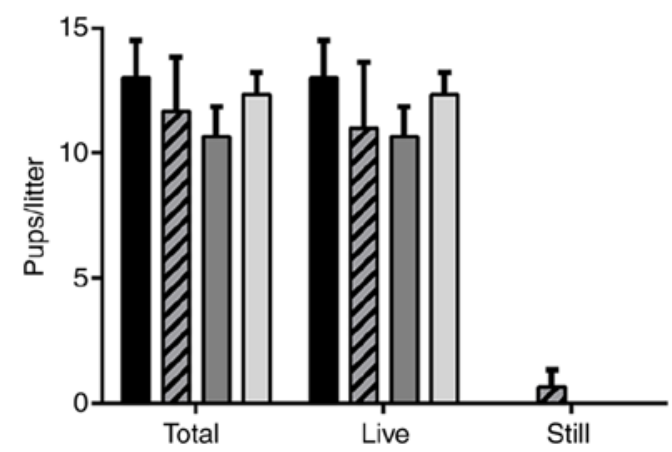

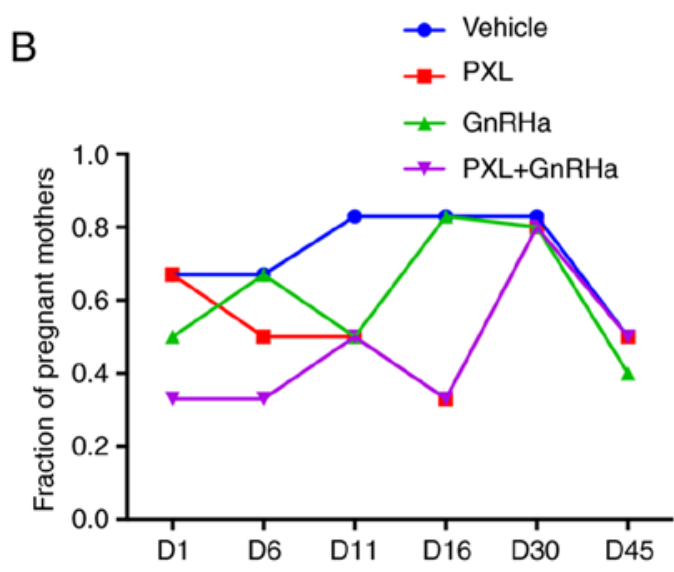

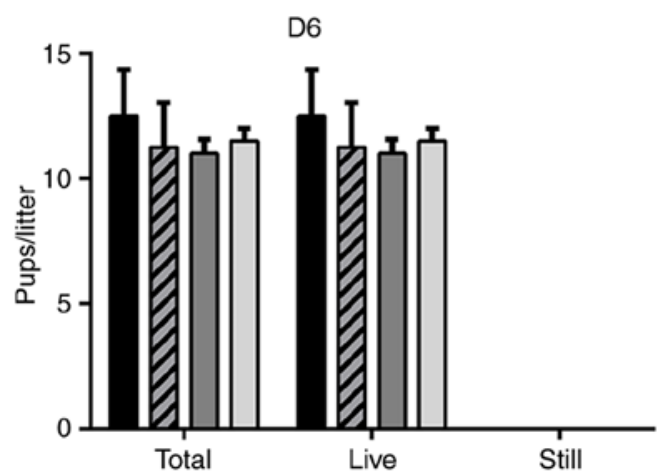

D16

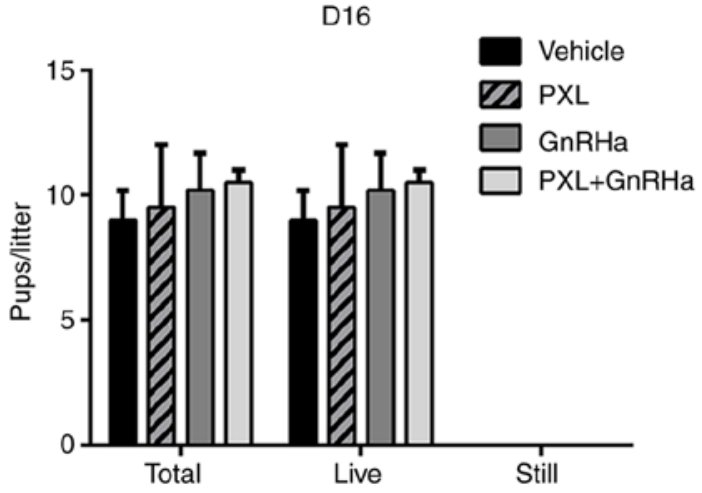

Figure 5. Adverse reproductive outcomes PXL-induced last for 1 estrous cycle, and GnRHa reduces stillbirths. (A) Live pups per litter delivered in the four groups on days 1-45 following chemotherapy. (B) Pregnancy rate in the 4 groups on days 1-45 following chemotherapy. (C) Total pups, live pups and stillbirths per litter delivered in the four groups days 1-16 following chemotherapy. Data are expressed as the mean \pm SEM. Statistical analysis was performed by one-way ANOVA followed by LSD post-hoc test. "P<0.05 vs. the control group (vehicle). PXL, paclitaxel; GnRHa, gonadotropin-releasing hormone agonist.

slightly higher than that in the PXL group, although without significance $(38.25 \pm 8.35$ vs. $18.00 \pm 6.49 ; \mathrm{P}=0.058)$. On days 11 and 16, the number and percentage of MII oocytes were similar among the four groups (Fig. 3).

Although some oocytes developed into MII oocytes, we cannot say that all of them were normal. Spindle morphology, $\mathrm{CH}$ alignment, karyotype analysis and in vitro fertilization were used to evaluate the quality of MII oocytes. Spindle apparatus immunostaining revealed that, on day 1 following chemotherapy, most oocytes collected from the control or GnRHa groups had typical barrel-like spindles with $\mathrm{CHs}$ located on the equatorial plate (Fig. 4C). By contrast, a majority of disorganized spindles and misaligned $\mathrm{CHs}$ were observed in the PXL group (spindle, $25.32 \pm 22.69 \%$ vs. $69.64 \pm 7.76 \%$;

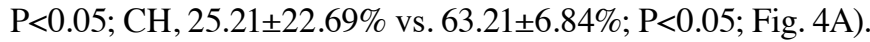
Co-treatment of GnRHa tended to protect spindle organization, although without significance (spindle, $57.50 \pm 18.12 \%$ vs. $25.32 \pm 22.69 \%$; $\mathrm{P}>0.05 ; \mathrm{CH}, 47.5 \pm 29.18 \%$ vs. $25.21 \% \pm 22.69 \%$; $\mathrm{P}>0.05$; Fig. 4A). A significantly higher frequency of aneuploid oocytes of \pm 20 univalents was found in the PXL group than in the control or GnRHa groups $(79.17 \pm 5.15 \%$ vs. $24.44 \pm 21.76 \%$ vs. $18.75 \pm 8.11 \%$; $\mathrm{P}<0.05$; Fig. $4 \mathrm{~B}$ ) on day 1 following chemotherapy. Co-treatment with PXL and GnRHa tended to reduce the aneuploidy rate $(79.17 \pm 5.15 \%$ vs. $45.00 \pm 5.03 \%$; $\mathrm{P}>0.05$; Fig. 4B). The fertilization rate was also clearly decreased in the PXL group, as compared with the control (27.27\% vs. $75.51 \%$; $\mathrm{P}<0.05$; Fig. 4D; Table SI) on day 1 following chemotherapy. The fertilization ability of the PXL+GnRHa group was also 
Table I. Reproductive outcomes of mice mated during the 1st estrous cycle after chemotherapy.

\begin{tabular}{|c|c|c|c|c|c|c|c|c|}
\hline Groups & $\mathrm{N}$ & Litters (\%) & $\begin{array}{l}\text { Total pups } \\
\text { (live pups) }\end{array}$ & $\begin{array}{c}\text { Live } \\
\text { pups/litter }\end{array}$ & P-value & Stillbirths & Stillbirths/litter ${ }^{\mathrm{a}}$ & P-value \\
\hline Vehicle & 6 & $4(66.7)$ & $50(48)$ & $12.00 \pm 2.16$ & & 2 & $0.50 \pm 0.50$ & \\
\hline PXL & 6 & $4(66.7)$ & $39(21)$ & $5.25 \pm 3.20$ & 0.066 & 18 & $4.50 \pm 2.10^{\mathrm{b}}$ & 0.050 \\
\hline GnRHa & 6 & $3(50.0)$ & $28(28)$ & $9.33 \pm 1.20$ & 0.464 & 0 & $0.00 \pm 0.00^{\mathrm{b}}$ & $0.799(0.042)$ \\
\hline PXL+GnRHa & 6 & $2(33.3)$ & $22(22)$ & $11.00 \pm 0.00$ & 0.806 & 0 & $0.00 \pm 0.00$ & 0.822 \\
\hline
\end{tabular}

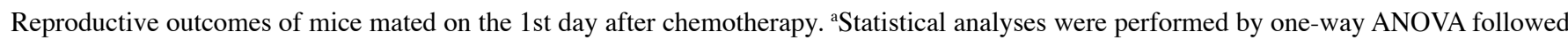

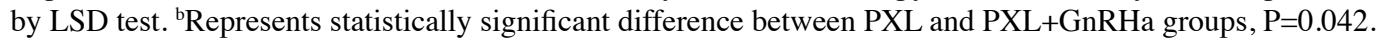

A
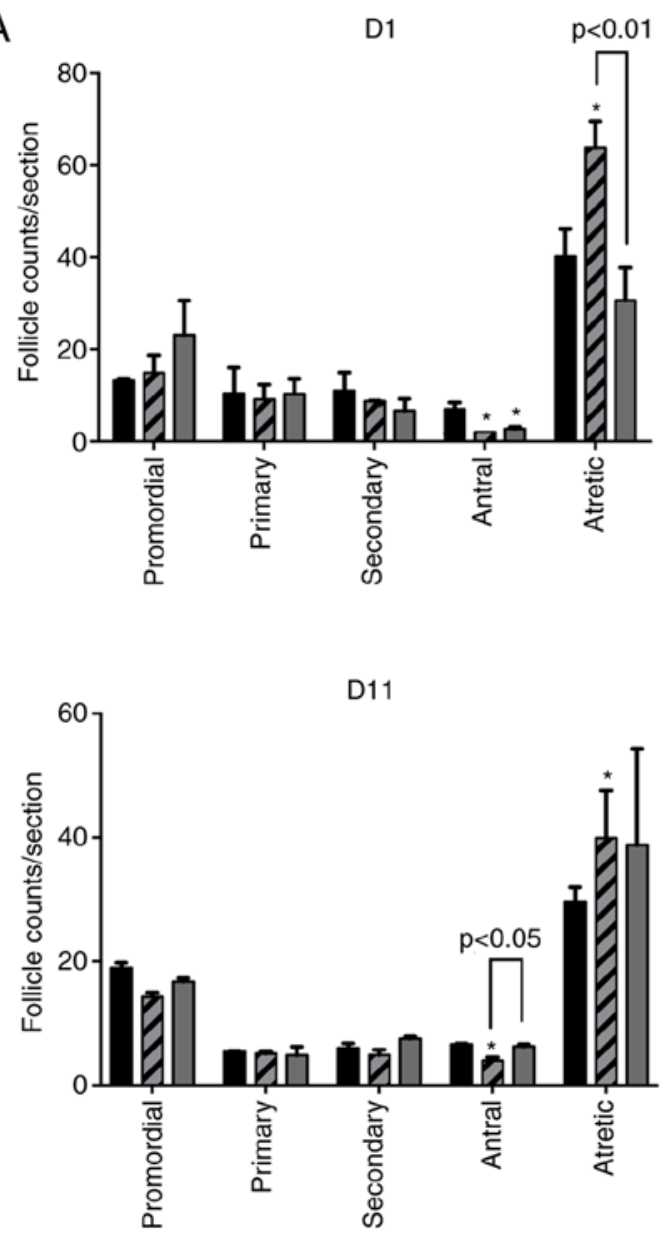
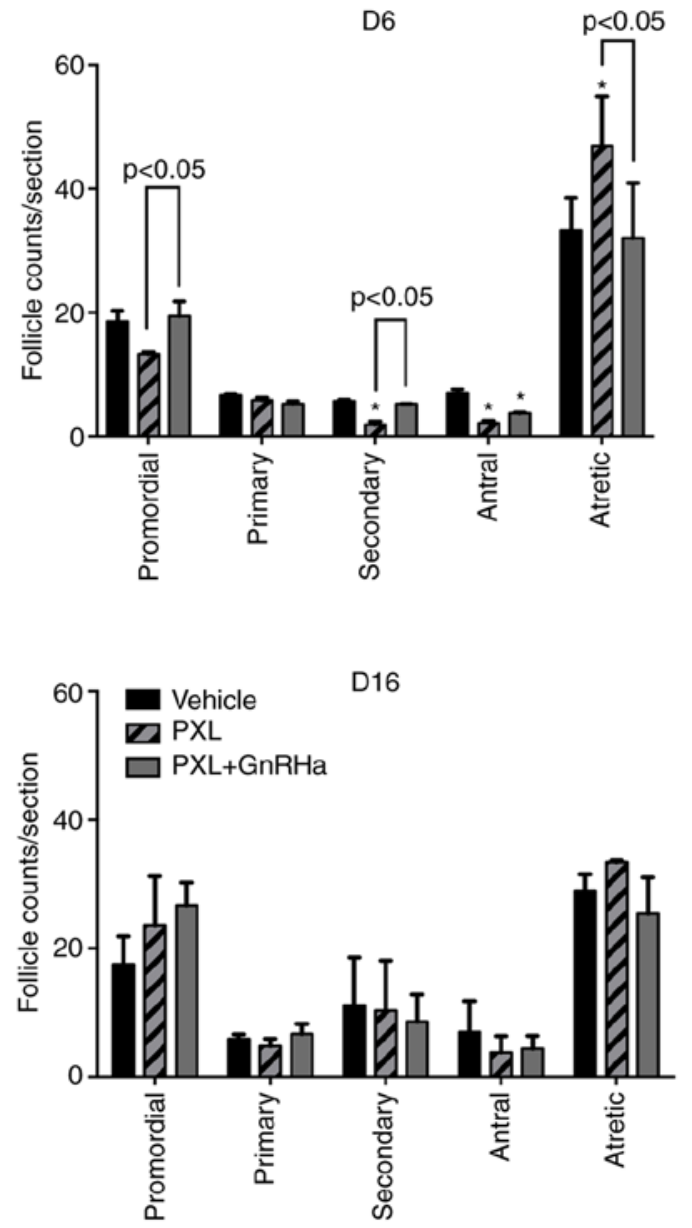

B

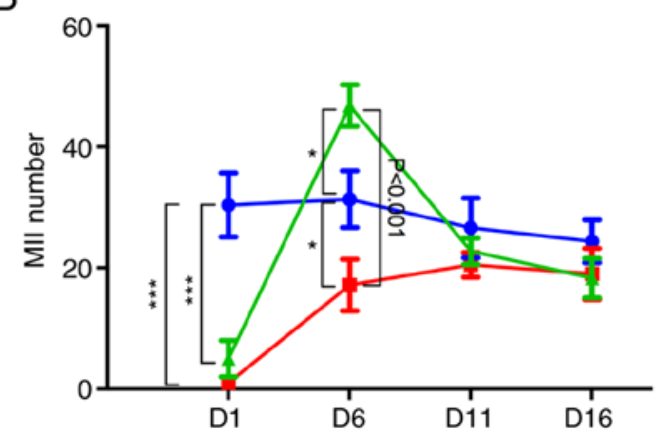

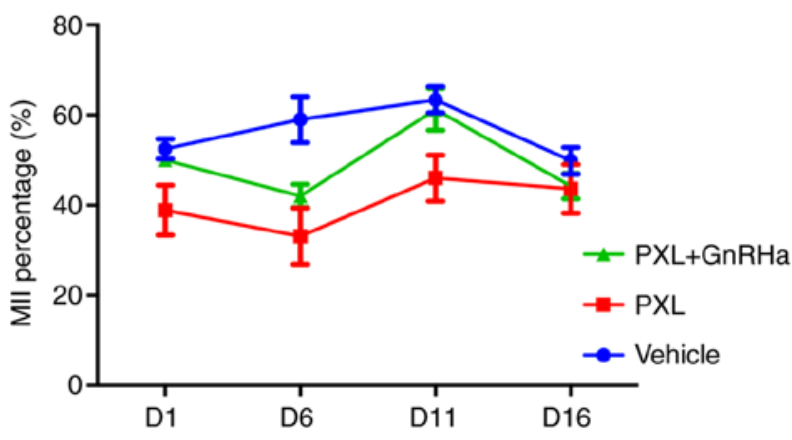

Figure 6. Multiple doses of PXL also damage antral follicles in a transient way. (A) Number of primordial, primary, secondary, antral and atretic follicles and (B) The number and percentage of MII oocytes retrieved in the three groups on days 1-16 after 3 doses of chemotherapy. Data are expressed as the mean \pm SEM. Statistical analysis was performed by one-way ANOVA followed by LSD test. ${ }^{*} \mathrm{P}<0.05,{ }^{* * * *} \mathrm{P}<0.001$ vs. the control group (vehicle). PXL, paclitaxel; MII, metaphase II; GnRHa, gonadotropin-releasing hormone agonist. 
partially restored (64.29 vs. $27.27 \%$; $\mathrm{P}>0.05$ ). After 1 estrous cycle, on day 6 following chemotherapy, the normal spindle morphology, as well as $\mathrm{CH}$ alignment, aneuploidy and fertilization rates of the oocytes in the PXL group had all reached the same level as those of the control group (Fig. 4).

PXL-induced adverse reproductive outcomes last for 1 estrous cycle and GnRHa reverses this outcome. A mating experiment was carried out to study whether damage to oocytes caused by PXL could affect reproductive outcomes. After mating on day 1 following chemotherapy, animals treated with GnRHa produced fewer litters (Table I; Fig. 5B), as 4/6 mice were pregnant in the control and PXL group, but only 2-3 mice in the GnRHa or PXL+GnRHa groups. No significant difference in live pups per litter was observed among the four groups. However, markedly, the PXL group delivered a total of 18 dead pups, which was more than those delivered in the control group $(\mathrm{P}=0.05)$ and the $\mathrm{GnRHa}$ group $(\mathrm{P}=0.046)$. There was no stillbirth in the PXL+GnRHa group but since only 2 mother mice delivered, no statistical difference was identified. (Table I; Fig. 5A and C). On day 6 and 11 following chemotherapy, the percentage of pregnant mothers in mice treated with GnRHa remained low. But on day 16, the pregnancy rate of mice treated with PXL dropped. As for live pups/litters and stillbirths, 1 estrous cycle after chemotherapy, the live pups/litters delivered in each group were similar, with stillbirth seldom happening (Table I; Fig. 5A and C). To ensure that the offspring of PXL-exposed animals had no potential genetic defects, we examined the karyotypes of 3 random pups from every pregnant mother. The results revealed that every live pup had a euploid karyotype (Tables SII-SV).

Multiple doses of PXL do not cause permanent damage to mouse fertility. In order to mimic the clinical usage of PXL, 3 subsequent doses of PXL were administered to mice (1 dose per 3 days). Since it was determined that GnRHa had no adverse effect on mouse fertility in the single-dose experiment, in order to reduce mice sacrificing in our study, we canceled this group in the multiple-dose experiment. The GnRHa was administered 1 estrous cycle before, during and continuing with one more estrous cycle following chemotherapy (Fig. 1B). The follicle counts were similar to those of the single-dose-PXL experiment. On day 1 following 3 doses of chemotherapy, the antral follicles in the PXL and PXL+GnRHa groups were significantly reduced $(7.00 \pm 1.00$ vs. $2.00 \pm 0.00$ vs. $2.70 \pm 0.30 ; \mathrm{P}<0.05)$. The primordial, primary and secondary follicles were all similar between groups. The administration of GnRHa during chemotherapy significantly reduced the PXL-induced atretic follicles $(30.60 \pm 5.00$ vs.

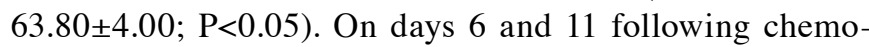
therapy, antral follicles in the PXL group were still less than those in the control, but co-treatment with PXL and GnRHa helped antral follicles recover on day 11. The increase in atretic follicles in the PXL group also lasted until day 11, but the PXL+GnRHa group maintained a similar amount of atretic follicles to that of the control group. On day 16 following chemotherapy, no significant difference in follicles of all stages was identified among groups (Fig. 6A). To further estimate the duration of the gonadotoxicity caused by multiple doses of PXL administration, ovarian stimulation was also performed. On days 1 and 6 following the last dose of PXL, significantly less MII oocytes were collected in the PXL group than in the control (D1, $1.00 \pm 0.00$ vs. $30.40 \pm 5.27 ; \mathrm{P}<0.001$; D6, $17.20 \pm 4.25$ vs. $31.33 \pm 4.67$; $\mathrm{P}<0.05$; Fig. $6 \mathrm{~B})$. With the protection of GnRHa, even more MII oocytes were retrieved than in the control $(46.80 \pm 3.44$ vs. $31.33 \pm 4.67 ; \mathrm{P}<0.05$; Fig. $6 \mathrm{~B})$ on day 6 following chemotherapy. On days 11 and 16, the amount of MII oocytes was similar in all groups.

\section{Discussion}

In the present study, it was determined that PXL only induced loss of antral follicles and increased atretic follicles without causing any loss of primordial, primary or secondary follicles. Notably, after 1-2 estrous cycles for recovery, the follicle counts of each stage tended to be the same among groups. In addition, AMH examination verified that the ovarian reserve was not affected by PXL. PXL only affected antral follicles. That was consistent with the results of former studies, which reported that PXL acted on cells with active division, irrespective of their malignancy (30), since the development from secondary to antral follicles is a process in which granulosa cells, theca cells and vessels proliferate rapidly (31). The transient impact of PXL may be due to its short plasma half-time (32-34) and limited effect on pre-antral follicles. When pre-antral follicles grew into antral follicles in the next estrous cycle, the plasma concentration of PXL had already decreased. As a result, after one estrous cycle, the morphology of follicles was able to recover.

To understand which stage of oocytes in antral-preovulatory follicles would be affected by PXL, an in vitro experiment was carried out. The results revealed that, following exposure to PXL, mice GV oocytes were capable of maturation, which suggested that GV oocytes were insensitive to PXL, since microtubules had not assembled into specific forms at this stage $(35,36)$. However, oocytes in MI or MII were very sensitive to PXL, as their development depends on the assembly of microtubules $(37,38)$ and the formation of a spindle apparatus, which were destroyed by exposure to PXL.

To confirm the effect of PXL on oocytes in vivo, an animal model was designed to estimate the time mice required to recover normal ovulation. This was measured through ovarian stimulation of mice at different time-points following chemotherapy. It was determined that PXL caused acute oocyte damage. Following the administration of PMSG in mice 1 day after chemotherapy, when oocytes were in antral-preovulatory follicles and directly exposed to PXL, the number and quality of oocytes was markedly decreased. Following 1 estrous cycle, MII oocytes collected in the PXL group had a similar quality to those of the control group. Two estrous cycles after chemotherapy, the MII oocytes in the PXL and control groups were totally comparable. These results were consistent with the transient effect of PXL on antral follicles, and supplemented the findings of a previous study, which stated that PXL caused a meiotic maturation delay and spindle defects in mouse oocytes on hCG trigger day (14).

The mating experiment indicated that, although PXL did not disrupt copulation, it could cause stillbirths, which was consistent with a previous study (18). Following 1 estrous cycle, the recovery of litter size and disappearance of stillbirth 
in the PXL group was consistent with the effect of PXL on antral follicles and oocytes, further verifying the transient effect of PXL in vivo. Of note, there was 1 stillbirth on day 11 in the PXL group, which may have been a random incident, since there was no other stillbirth in any subsequent mating; 3 stillbirths were recorded in the control group on day 1 . The karyotypes of every live pup delivered in all four groups were normal, which indicated that PXL caused lethal impairment in oocytes. On day 16, a slope in the pregnancy rate was observed in the PXL group, following repeated experiments. However, on days 30 and 45 following chemotherapy, the pregnancy rate and litter size in the PXL group were similar to those of the control group. This result suggested that the reduced pregnancy rate in the PXL group on day 16 was not due to the decrease in ovarian reserve, but other unknown reasons that require further exploration.

GnRHa have been studied as protective agents of chemotherapy-induced ovarian failure for decades. The mechanism of GnRHa was considered to be decreasing FSH levels and suppressing follicle growth through the pituitary-gonadal axis (39), as well as upregulating anti-apoptotic molecules (40) and decreasing the exposure of primordial follicles to cytotoxic agents (41). However due to contradictory results of clinical trials $(23,42)$, to date, GnRHa has not been recommended as a regular ovarian protective agent $(43,44)$. In the present study, the follicle count results revealed that GnRHa suppressed follicle maturation effectively. GnRHa reduced atretic follicles in the PXL+GnRHa group, which suggested that pretreatment with GnRHa kept ovaries in a relatively quiescent condition to avoid damage induced by PXL to antral follicles. It was speculated that the growing follicles were suppressed due to the administration of $\mathrm{GnRHa}$ before they became sensitive to PXL. During PMSG or mating-induced ovulation when the plasma concentration of PXL decreased rapidly, exposure to PXL at lower concentrations led to the better-quality of oocytes. In the mating experiment, mice that received a GnRHa injection delivered fewer litters, since GnRHa disrupted copulation by suppressing reproductive hormones. In combination, the protective effect of GnRHa was not obvious, possibly due to the short duration of the effect of PXL on ovaries.

Multiple-dose administration of PXL had a similar effect on ovaries to that of the single-dose experiment, suggesting that there was no cumulative effect or follicle exhaustion caused by PXL. This mouse model mimicked the common clinical chemotherapy regimen with PXL included [i.e., TP regimen in ovarian cancer (45) and AC-T regimen in breast cancer (11), in which multiple courses of PXL treatment are required]. Notably, ovulation in the PXL+GnRHa group was higher than that of the control 1 estrous cycle after the last dose of PXL. This may be due to the controlled ovarian hyperstimulation effect of GnRHa followed by gonadotrophin PMSG. This suggested that the temporary destruction of the oocytes was more likely associated with the last dose of PXL, which once again underlined the transient effect of PXL on ovaries.

In clinical practice, physicians may face several challenges, such as what the required duration of contraceptive method administration is following chemotherapy and whether GnRHa should be used to protect ovarian function. Physicians used to recommend 6 months of contraceptives based on experience. The present study revealed that PXL had no impact on ovarian reserve and only a transient one on oocytes. The present laboratory evidence provided the possibility for shortening contraceptive method administration following PXL chemotherapy. The present study also revealed that GnRHa has a protective effect on ovaries in PXL chemotherapy, and provided a scheme of GnRHa administration for reproductive protection, which suggests that the application of GnRHa should be encouraged in PXL-based chemotherapeutic regimens. Further research should be carried out to determine whether the impact of PXL on human ovaries and oocytes is consistent with its impact on mouse ovaries and oocytes.

\section{Acknowledgements}

Not applicable.

\section{Funding}

The present study was supported by the National Key Technology R\&D Program of China (grant no. 2019YFC1005200, 2019YFC1005202 and 2018YFC1002103), National Natural Science Foundation of China (grant no. 81802896), the Natural Science Foundation of Hubei Province (grant no. 2017CFB800) and the Hubei Province Health and Family Planning Scientific Research Project (grant nos. WJ2017Z013 and WJ2019M127).

\section{Availability of data and materials}

The datasets used and/or analyzed during the present study are available from the corresponding author on reasonable request.

\section{Authors' contributions}

NM performed the experiments, analyzed and interpreted the data and wrote the manuscript. $\mathrm{GeC}$ contributed to the execution of the oocyte experiment. JC performed the hormone assays and assisted in data analysis. MC performed the animal experiments and acquired the data. YY performed the histological analyses of ovaries. QL, MT and XF assisted in the animal experiments. XL contributed to the designing and drafting the article and discussed the results. SZ contributed to the analysis and interpretation of data. $\mathrm{DM}$ and $\mathrm{GaC}$ conceived the study, discussed the results and supervised the study. KL conceived the study, designed the experiments, conducted study, discussed the results, contributed to the drafting of the article, submission and revision. JA conceived the study, designed the experiments, discussed the results and supervised the study. All the authors have read and approved the final article.

\section{Ethics approval and consent to participate}

All experiments were approved by the Institutional Ethics Committee of Tongji Hospital, Tongji Medical College, Huazhong University of Science and Technology (approval no. TJ-A20161101).

\section{Patient consent for publication}

Not applicable. 


\section{Competing interest}

The authors declare that they have no competing interests.

\section{References}

1. Olorunfemi G, Ndlovu N, Masukume G, Chikandiwa A, Pisa PT and Singh E: Temporal trends in the epidemiology of cervical cancer in South Africa (1994-2012). Int J Cancer 143: 2238-2249, 2018.

2. Brinton LA, Sherman ME, Carreon JD and Anderson WF: Recent trends in breast cancer among younger women in the United States. J Natl Cancer Inst 100: 1643-1648, 2008.

3. Huang Z, Zheng Y, Wen W, Wu C, Bao P, Wang C, Zhong W, Gao YT, Jin F, Xiang YB, et al: Incidence and mortality of gynaecological cancers: Secular trends in urban Shanghai, China over 40 years. Eur J Cancer 63: 1-10, 2016.

4. Gomez SL, Von Behren J, McKinley M, Clarke CA, ShariffMarco S, Cheng I, Reynolds P and Glaser SL: Breast cancer in Asian Americans in California, 1988-2013: Increasing incidence trends and recent data on breast cancer subtypes. Breast Cancer Res Treat 164: 139-147, 2017

5. Aareleid T, Zimmermann ML, Baburin A and Innos K: Divergent trends in lung cancer incidence by gender, age and histological type in Estonia: A nationwide population-based study. BMC Cancer 17: 596, 2017.

6. Siegel RL, Miller KD and Jemal A: Cancer statistics, 2018. CA Cancer J Clin 68: 7-30, 2018.

7. Roness H, Kashi $\mathrm{O}$ and Meirow D: Prevention of chemotherapy-induced ovarian damage. Fertil Steril 105: 20-29, 2016.

8. Donnez J and Dolmans MM: Fertility preservation in women. N Engl J Med 377: 1657-1665, 2017.

9. Sofias AM, Dunne M, Storm G and Allen C: The battle of 'nano' paclitaxel. Adv Drug Deliv Rev 122: 20-30, 2017.

10. Koh WJ, Abu-Rustum NR, Bean S, Bradley K, Campos SM, Cho KR, Chon HK, Chu C, Clark R, Cohn D, et al: Cervical cancer, version 3.2019, NCCN clinical practice guidelines in oncology. J Natl Compr Canc Netw 17: 64-84, 2019.

11. Gradishar WJ, Anderson BO, Abraham J, Aft R, Agnese D, Allison KH, Blair SL, Burstein HJ, Dang C, Elias AD, et al: Breast cancer, version 3.2020, NCCN clinical practice guidelines in oncology. J Natl Compr Canc Netw 18: 452-478, 2020.

12. Ettinger DS, Wood DE, Aggarwal C, Aisner DL, Akerley W, Bauman JR, Bharat A, Bruno DS, Chang JY, Chirieac LR, et al: NCCN guidelines insights: Non-small cell lung cancer, version 1.2020. J Natl Compr Canc Netw 17: 1464-1472, 2019.

13. Alushin GM, Lander GC, Kellogg EH, Zhang R, Baker D and Nogales E: High-resolution microtubule structures reveal the structural transitions in $\alpha \beta$-tubulin upon GTP hydrolysis. Cell 157: 1117-1129, 2014.

14. Mailhes JB, Carabatsos MJ, Young D, London SN, Bell M and Albertini DF: Taxol-induced meiotic maturation delay, spindle defects, and aneuploidy in mouse oocytes and zygotes. Mutat Res 423: 79-90, 1999.

15. Ozcelik B, Turkyilmaz C, Ozgun MT, Serin IS, Batukan C, Ozdamar S and Ozturk A: Prevention of paclitaxel and cisplatin induced ovarian damage in rats by a gonadotropin-releasing hormone agonist. Fertil Steril 93: 1609-1614, 2010

16. Gücer F, Balkanli-Kaplan P, Doganay 1, Yüce MA, Demiralay E, Sayin NC and Yardim T: Effect of paclitaxel on primordial follicular reserve in mice. Fertil Steril 76: 628-629, 2001.

17. Tarumi W, Suzuki N, Takahashi N, Kobayashi Y, Kiguchi K, Sato K and Ishizuka B: Ovarian toxicity of paclitaxel and effect on fertility in the rat. J Obstet Gynaecol Res 35: 414-420, 2009.

18. Kai S, Kohmura H, Hiraiwa E, Koizumi S, Ishikawa K, Kawano S, Kuroyanagi $\mathrm{K}$, Hattori $\mathrm{N}$, Chikazawa $\mathrm{H}$, Kondoh $\mathrm{H}$, et al: Reproductive and developmental toxicity studies of paclitaxel. (I)-Intravenous administration to rats prior to and in the early stages of pregnancy. J Toxicol Sci 19 (Suppl 1): S57-S67, 1994 (In Japanese).

19. Reh A, Oktem O and Oktay K: Impact of breast cancer chemotherapy on ovarian reserve: A prospective observational analysis by menstrual history and ovarian reserve markers. Fertil Steril 90: 1635-1639, 2008.

20. Zhao J, Liu J, Chen K, Li S, Wang Y, Yang Y, Deng H, Jia W, Rao N, Liu Q and Su F: What lies behind chemotherapy-induced amenorrhea for breast cancer patients: A meta-analysis. Breast Cancer Res Treat 145: 113-128, 2014.
21. Hamy AS, Porcher R, Eskenazi S, Cuvier C, Giacchetti S, Coussy F, Hocini H, Tournant B, Perret F, Bonfils S, et al: Anti-mullerian hormone in breast cancer patients treated with chemotherapy: A retrospective evaluation of subsequent pregnancies. Reprod Biomed Online 32: 299-307, 2016.

22. Sukumvanich P, Case LD, Van Zee K, Singletary SE, Paskett ED, Petrek JA, Naftalis E and Naughton MJ: Incidence and time course of bleeding after long-term amenorrhea after breast cancer treatment: A prospective study. Cancer 116: 3102-3111, 2010.

23. Silva C, Caramelo O, Almeida-Santos T and Ribeiro Rama AC: Factors associated with ovarian function recovery after chemotherapy for breast cancer: A systematic review and meta-analysis. Hum Reprod 31: 2737-2749, 2016.

24. Senra JC, Roque M, Talim MCT, Reis FM and Tavares RLC: Gonadotropin-releasing hormone agonists for ovarian protection during cancer chemotherapy: Systematic review and meta-analysis. Ultrasound Obstet Gynecol 51: 77-86, 2018.

25. Munhoz RR, Pereira AA, Sasse AD, Hoff PM, Traina TA, Hudis CA and Marques RJ: Gonadotropin-releasing hormone agonists for ovarian function preservation in premenopausal women undergoing chemotherapy for early-stage breast cancer: A systematic review and meta-analysis. JAMA Oncol 2: 65-73, 2016.

26. National Institutes of Health (NIH), Animal Research Advisory Committee: NIH Guidelines for Endpoints in Animal Study Proposals. NIH, Bethesda, MD, 2016.

27. Wang XN, Roy SK and Greenwald GS: In vitro DNA synthesis by isolated preantral to preovulatory follicles from the cyclic mouse. Biol Reprod 44: 857-863, 1991

28. Cardiff RD, Miller CH and Munn RJ: Manual hematoxylin and eosin staining of mouse tissue sections. Cold Spring Harb Protoc 2014: 655-658, 2014

29. Myers M, Britt KL, Wreford NG, Ebling FJ and Kerr JB: Methods for quantifying follicular numbers within the mouse ovary. Reproduction 127: 569-580, 2004.

30. Tinwell $\mathrm{H}$ and Ashby $\mathrm{J}$ : Genetic toxicity and potential carcinogenicity of taxol. Carcinogenesis 15: 1499-1501, 1994.

31. Oktem $\mathrm{O}$ and Urman B: Understanding follicle growth in vivo. Hum Reprod 25: 2944-2954, 2010.

32. Rezazadeh M, Emam J, Mostafavi A, Rostami M, Hassanzadeh F, Sadeghi H, Minaiyan M and Lavasanifar A: A rapid and sensitive HPLC method for quantitation of paclitaxel in biological samples using liquid-liquid extraction and UV detection. Application to pharmacokinetics and tissues distribution study of paclitaxel loaded targeted polymeric micelles in tumor bearing mice. J Pharm Pharm Sci 18: 647-660, 2015.

33. Kim JE and Park YJ: Paclitaxel-loaded hyaluronan solid nanoemulsions for enhanced treatment efficacy in ovarian cancer. Int J Nanomedicine 12: 645-658, 2017.

34. Anwar M, Akhter S, Mallick N, Mohapatra S, Zafar S, Rizvi MMA, Ali A and Ahmad FJ: Enhanced anti-tumor efficacy of paclitaxel with PEGylated lipidic nanocapsules in presence of curcumin and poloxamer: In vitro and in vivo studies. Pharmacol Res 113 (Pt A): 146-165, 2016.

35. Coticchio G, Dal Canto M, Mignini Renzini M, Guglielmo MC, Brambillasca F, Turchi D, Novara PV and Fadini R: Oocyte maturation: Gamete-somatic cells interactions, meiotic resumption, cytoskeletal dynamics and cytoplasmic reorganization. Hum Reprod Update 21: 427-454, 2015.

36. Oktem O and Oktay K: The ovary: Anatomy and function throughout human life. Ann N Y Acad Sci 1127: 1-9, 2008.

37. Capalbo A, Hoffmann ER, Cimadomo D, Ubaldi FM and Rienzi L: Human female meiosis revised: New insights into the mechanisms of chromosome segregation and aneuploidies from advanced genomics and time-lapse imaging. Hum Reprod Update 23: 706-722, 2017.

38. Sun QY, Lai L, Wu GM, Park KW, Day BN, Prather RS and Schatten H: Microtubule assembly after treatment of pig oocytes with taxol correlation with chromosomes, gamma-tubulin, and MAP Kinase. Mol Reprod Dev 60: 481-490, 2001.

39. Blumenfeld $\mathrm{Z}$ and von Wolff M: GnRH-analogues and oral contraceptives for fertility preservation in women during chemotherapy. Hum Reprod Update 14: 543-552, 2008.

40. Meirow D, Dor J, Kaufman B, Shrim A, Rabinovici J, Schiff E, Raanani H, Levron J and Fridman E: Cortical fibrosis and bloodvessels damage in human ovaries exposed to chemotherapy. Potential mechanisms of ovarian injury. Hum Reprod 22: 1626-1633, 2007.

41. Blumenfeld Z: How to preserve fertility in young women exposed to chemotherapy? The role of GnRH agonist cotreatment in addition to cryopreservation of embrya, oocytes, or ovaries. Oncologist 12: 1044-1054, 2007. 
42. Elgindy EA, El-Haieg DO, Khorshid OM, Ismail EI, Abdelgawad M, Sallam HN and Abou-Setta AM: Gonadatrophin suppression to prevent chemotherapy-induced ovarian damage: A randomized controlled trial. Obstet Gynecol 121: 78-86, 2013.

43. Ethics Committee of American Society for Reproductive Medicine: Fertility preservation and reproduction in patients facing gonadotoxic therapies: A committee opinion. Fertil Steril 100: 1224-1231, 2013.

44. Loren AW, Mangu PB, Beck LN, Brennan L, Magdalinski AJ, Partridge AH, Quinn G, Wallace WH and Oktay K; American Society of Clinical Oncology: Fertility preservation for patients with cancer: American society of clinical oncology clinical practice guideline update. J Clin Oncol 31: 2500-2510, 2013.
45. Morgan RJ Jr, Armstrong DK, Alvarez RD, Bakkum-Gamez JN, Behbakht K, Chen LM, Copeland L, Crispens MA, DeRosa M, Dorigo O, et al: Ovarian cancer, version 1.2016, NCCN clinical practice guidelines in oncology. J Natl Compr Canc Netw 14: 1134-1163, 2016

(i) $\Theta$ This work is licensed under a Creative Commons Attribution-NonCommercial-NoDerivatives 4.0 International (CC BY-NC-ND 4.0) License. 